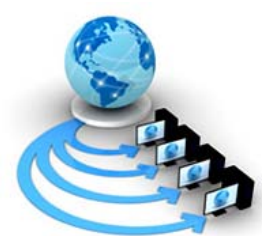

Volume 10, No. 3, May-June 2019

International Journal of Advanced Research in Computer Science

REVIEW ARTICLE

Available Online at www.ijarcs.info

\title{
ENERGY EFFICIENT LEACH AND IMPROVED LEACH: A REVIEW
}

\author{
Preeti \\ Department of computer science and engineering \\ Tulas Institute, Uttrakhand Technical University \\ Dehradun, India
}

\author{
Monika Belwal \\ Department of computer science and engineering \\ Tulas Institute, Uttrakhand Technical University \\ Dehradun, India
}

\begin{abstract}
Energy Efficiency is the most crucial requirement of sensor nodes in WSN networks. Sensor nodes are the communicating nodes of wireless network which lost their energies with package of time due to having small battery life. So, for sustaining the wireless network to work for longer period of time there requires the need of energy efficient routing protocols. Here in this paper we are presenting LEACH (Low Energy Adaptive Clustering Hierarchy) and PEGASIS (Power Efficient Gathering in Sensor Information System) protocols as most energy efficient protocols to prolong the higher lifetime and small delay time of WSN network.
\end{abstract}

Keywords: LEACH, PEGASIS, WSN, MATLABR2010b, Hierarchical Clustering, Cluster Head(CH), Chain routing.

\section{INTRODUCTION}

WSN refers to wireless sensor nodes in which the sensor nodes work as key players to establish the wireless communication. These nodes are geographically distributed in the network and communicate with one another and to base station to maintain the communication. Sensor nodes are made up of battery, microcontroller, sensor and memory units [3] which are prone to energy related issues due to short battery life. So, using of routing protocols which are highly energy efficient is the key and vital requirement of a robust wireless sensor network.

Basically, WSN routing protocols can be categorizing as Flat, Hierarchical and Location based [4]. Our area of study moves around Hierarchical based routing protocols. We find that number of hierarchical based routing protocols like TEEN, APTEEN, LEACH, HEED, PEGASIS, VGA, MECN\&SMECN [3][4] are present.

By extensive literature review we find that two protocols LEACH and PEGASIS provides ideal paradigm for construction of highly energy efficient wireless network by increasing the lifetime of sensor nodes by optimizing the clustering process among communicating nodes. Both are categorizing as hierarchical Based Routing Protocols and works on data aggregation and fusion algorithms to deploy and reached sensed data from sensors to Base Station [4].

\section{LEACH}

LEACH is defined as Low Energy Adaptive Clustering Hierarchy protocol. It is a clustering based protocol that works on principle of data aggregation on to the cluster head by sensor nodes and reduces number of transmission on to the base station again and again by communicating nodes [5]. It works using TDMA/CDMA transmission technique to reduce collisions among nodes which further reduce the communication overheads by individual nodes to Base station and increases the amount of data transmission by accumulating data on to cluster head only and its transmission to base station in one move in allocated time frame. In $\mathrm{LEACH}$ all the nodes are grouped together to form a cluster and in each cluster one node is declared as cluster head $(\mathrm{CH})$ [3][4]. $\mathrm{CH}$ collects data from communicating nodes in the cluster and then sends the aggregated data to base station or sink in assigned time slot. Clusters are made dynamically using geographical distribution of the nodes and cluster head is created using shortest distance algorithm by computing the least distance of

Node to base station which become the next cluster head for next communication cycle. In this way each node becomes $\mathrm{CH}$ in its life cycle [3]. Each LEACH operation is followed by two basic operations Setup phase operation and Steady phase operation. Setup phase operation is used to create the cluster and to choose a cluster head $(\mathrm{CH})$ for corresponding cluster. Steady phase is used for transmission of aggregated information by the cluster head $(\mathrm{CH})$ to the Base station/Sink [3].

\section{LEACH ALGORITHM}

The algorithm works with four major steps [3]: -

1. Geographical Formation of Cluster using initial or residual energy as $E_{\text {in }}(n)$ of nodes.

2. Selection of Cluster Head in each cluster by calculating the distance d (n) of each node to base station.

3. Data Aggregation by cluster head using data fusion by communicating nodes.

4. Data Transmission done by communicating of Cluster Head with Base station.

After the transmission phase of each cycle maximum energy of each round is estimated using formula:

$\max \left(\mathrm{E}_{\text {in }}(\mathrm{n})-\mathrm{E}_{\mathrm{amp}} * \mathrm{k}^{*} \mathrm{~d}^{2}\right.$.

5 . On basis of step 2, 4 the selection of next cluster head is made.

\section{PEGASIS}

PEGASIS stands for power efficient gathering in sensor information system [1, 2]. It is a near optimal hierarchical chain based protocol that uses greedy method and is considered as an improvement over LEACH in increasing energy efficiency and lifetime of network. In this protocol each sensor node communicates with its neighboring node for 
data transmission and for data receiving in a chain fashion. There is no separate data aggregation takes place at header node instead data fusion takes place simultaneously at each communicating sensor node. Once complete data packet is received by header node it is processed and transferred directly to Base station (BS). Each node becomes a header node in its life span so takes turn to send data to the base station at least once in its lifetime.

The chain is created using procedure called chain construction and Data gathering and transmission. The chain is created using lattermost node from base station and its nearest neighbor as its next node and so on. The last node is base station and node before base station is considered ad header node [5]. Data aggregation and processing both takes place at header node which send the processed data to base station at the end. The communication process work on few assumptions that:

1. All the communicating nodes have prior global knowledge of the network [5].

2. The senor nodes are homogeneous and have uniform distribution of energy [1].

3. The base station is fixed not dynamic.

4. Transmitting energy cost depends upon the distance covered.

5. The chain is created using greedy algorithm.

PEGASIS helps each communicating node to use its minimum energy to send data to its next neighbor not to cluster head as in LEACH and thus achieve a 2 and 8 improvement factor as compared to LEACH and Direct transmission method $[1,5,6]$.

\section{PEGASIS ALGORITHM}

\section{The algorithm works with two major steps: -}

1. Chain Construction Phase in which most distant sensor node to base station is considered as starting node for chain construction and its next nearest node as next node of chain and so on using greedy approach the chain is created. The greedy approach helps to create the optimum length chain at which no node become dead depending upon energy values and continuous communication takes place during round of communication.

2. Data Gathering Phase in which head node is selected randomly and all the fused data by sensor nodes get accumulated by header node and then get transferred to base station.

If ' $R$ ' is node count for a round ' $p$ ' then head node will be $p$ $\bmod \mathrm{R}$.

\section{COMPARATIVE STUDY}

- As nodes in PEGASIS contact to its neighbors only, so transmitting energy and dissipation energy used is very less as compared to LEACH where each node contact to the cluster head instead of their distance to $\mathrm{CH}$.

- $\quad$ Since each node becomes head node in its life time in PEGASIS so there is load balancing in the chain and dissipated energy is also balanced whereas LEACH undergoes with unbalanced dissipation of energies of sensor nodes due to different distance values to $\mathrm{CH}$. The node which is close to $\mathrm{CH}$ has less dissipation instead of node which is further to $\mathrm{CH}$.
- In PEGASIS when a head node is elected its energy values and distance to $\mathrm{BS}$ is not taken into consideration which sometimes make data loss if that nodes become dead during communication whereas in LEACH the energy values of the $\mathrm{CH}$ should be maintained up to a threshold value and node which has less energy value than threshold value can never be elected as $\mathrm{CH}$. So, LEACH sometimes provides more robust configuration than PEGASIS in this extent.

- PEGASIS systems undergoes redundancy of information as head node is the only responsible source for data transmission whereas LEACH provides less redundant data due to more than one cluster head in one round of communication.

- Simulation results using Matlab R2010b simulator shows that PEGASIS provides better network lifetime and better energy efficient system as compared to LEACH. It shows about 2 and 8 improvement factor as compared to LEACH and direct transmission method.

\section{CONCLUSION AND FUTURE WORK}

We find that PEGASIS perform better than LEACH in term of energy conservation of nodes and prolonging lifetime of network. It limits number of transmission and receiving of information among communicating nodes. Nodes need to communicate to their nearest neighbors only and communication is made by head node to base station which involves less dissipation of energy as compared to LEACH. Also it eliminates the overhead of cluster formation again and again in a round of communication as made in case of LEACH.

But, PEGASIS also need some improvements in fields like consideration of energy values of nodes and distance of head node to the base station for selection of head node. Also, in place of greedy method some more advanced methods can be used for creation of long chain of nodes when number of nodes is high. Also, if head node dies whole data and information will be elapsed and difficult to retrieve. I think some future work can be done in this direction for making PEGASIS more strong and robust for creating more secured wireless network system.

\section{REFRENCES}

[1] Stephanie Lindsey and Cauligi S. Raghvendra , "PEGASIS: Power-Efficient Gathering in sensor information system". In processing of IEEE Aerospace Conference, April 2002.

[2] Payal Jain, Dr. Anu Chaudhary ," The comparison between LEACH protocol and PEGASIS protocol based on lifetime of wireless sensor network". IJCSMC, Vol.6,Issue 12,December 2017.

[3] Lalita Yadav,Ch. Sunitha,"Low Energy Adaptive Clustering Hierarchy in Wireless Sensor Network . IJCSIT, Vol. 3, 2014. 
[4] Vandana Sharma, Payal Jain ,’Various Hierarchical Routing Protocols in Wireless Sensor Network : A Survey ". IJCSMC,Vol.2,Issue 5,May 2013.

[5] Sujata,Brijbhusan,”Energy Efficient PEGASIS Routing Protocol in Wireless Sensor Network”. IRJET,Vol.4,Issue 07,July 2017.
[6] A presentation shared by Reena Shekar, https://www.slideshare.net/ReenaShekar/leach-pegasis.

[7] Alexandros-Nikolaos Zattas,"Energy-Efficient Protocols in Wireless Sensor Networks",University of Peloponnese ,2018 Greece. 